\title{
Small-scale spatial structure in plankton distributions
}

\author{
A. Tzella and P. H. Haynes \\ Department of Applied Mathematics and Theoretical Physics, University of Cambridge, UK
}

Received: 31 October 2006 - Published in Biogeosciences Discuss.: 4 December 2006

Revised: 21 February 2007 - Accepted: 1 March 2007 - Published: 2 March 2007

\begin{abstract}
The observed filamental nature of plankton populations suggests that stirring plays an important role in determining their spatial structure. If diffusive mixing is neglected, the various interacting biological species within a fluid parcel are determined by the parcel time history. The induced spatial structure has been shown to be a result of competition between the time evolution of the biological processes involved and the stirring induced by the flow as measured, for example, by the rate of divergence of the distance of neighbouring fluid parcels. In the work presented here we examine a simple biological model based on delaydifferential equations, previously seen in Abraham (1998), including nutrients, phytoplankton and zooplankton, coupled to a strain flow. Previous theoretical investigations made on a differential equation model (Hernández-Garcia et al., 2002) imply that the latter two should share the same small-scale structure. The generalisation from differential equations to delay-differential equations, associated with the addition of a maturation time to the zooplankton growth, should not make a difference, provided sufficiently small spatial scales are considered. However, this theoretical prediction is in contradiction with the results of Abraham (1998), where the phytoplankton and zooplankton structures remain uncorrelated at all length scales. A new set of numerical experiments is performed here which show that these two regimes coexist. On larger scales, there is a decoupling of the spatial structure of the zooplankton distribution on the one hand, and the phytoplankton and nutrient on the other. On the other hand, at small enough length scales, the phytoplankton and zooplankton share the same spatial structure as expected by the theory involving no maturation time.
\end{abstract}

Correspondence to: A. Tzella

(a.tzella@damtp.cam.ac.uk)

\section{Introduction}

The generation of patchiness in planktonic distributions is a result of the biological interactions between species coupled to the background fluid motion. Phytoplankton distributions during springtime blooms, a consequence of ocean stratification and seasonal increase in sunlight, are strongly inhomogeneous and filamental with structures that range from 1 to $100 \mathrm{~km}$. Moreover, these distributions exhibit similar power law spectra to physical quantities like sea surface temperature (Seuront et al., 1996, 1999). On the other hand, most observations of patchiness in zooplankton indicate a flatter or noisier spectrum than the phytoplankton (Tsuda et al., 1993), though this result has been questioned by Martin and Srokosz (2002). For a review see Martin (2003).

The phytoplankton filaments observed in colour satellite images are mostly formed in the strain-dominated regions of the ocean between mesoscale eddies or along fronts. At these scales, oceanic turbulence is now understood to be strongly anisotropic (McWilliams et al., 1994), dominated by the directional activity of these eddies and fronts. Consequently, models employing eddy diffusion in order to parametrise turbulence and explain patchiness (Okubo, 1971) are rendered irrelevant at the mesoscale. Instead, it is advection which plays an important role in their formation.

Unlike eddy diffusion, advection is responsible for the transfer of large-scale inhomogeneities into smaller scales. Such a transfer from the larger scales $(\sim 100 \mathrm{~km})$ has been shown numerically to take approximately 10 days to reach $\sim 1 \mathrm{~km}$ at which point three-dimensional flow becomes important (Klein and Hua, 1990). This is less than the maturation time of zooplankton such as copepods which is typically 25 days (Kiørboe and Sabatini, 1995). This means that during their lifetime, any large-scale variation (e.g. $\sim 100 \mathrm{~km}$ ) will be stirred down to kilometre lengths.

It has been previously recognised (e.g. Haynes, 1999) that the dominant contribution to advection in large scale stably

Published by Copernicus GmbH on behalf of the European Geosciences Union. 
stratified geophysical flows can be successfully captured by two-dimensional spatially smooth and time-dependent velocity fields, that generate chaotic trajectories for the fluid particles. This chaotic advection (Aref, 1984) leads to smallscale structures in inert tracers. It turns out that a number of explicit results are insensitive to the model's details. It is sufficient to know the ability of a flow to mix with a tracer, measured by the rate at which fluid parcel trajectories diverge from each other (Ottino, 1989). At scales larger than the mixed-layer turbulence scale of a few hundred metres the effects of molecular diffusion can be neglected and the biological properties within a fluid parcel can be considered to evolve independently of its surroundings. Hence, the concentrations of different planktonic species can be taken to be uniformly distributed within a fluid parcel and determined by the time history of that parcel.

The emergence of persistent patterns requires some spatially varying external forcing such as a localised upwelling of nutrients or a latitudinal variation of sunlight. The induced spatial structure has been shown to be a result of competition between the rate of convergence of the biological processes involved and the rate of divergence of the distance of neighbouring fluid parcels. It has also been argued that, except under rather special conditions, the small scale behaviour should be the same for all interacting species (Neufeld et al., 1999; Hernández-Garcia et al., 2001, 2002). On the other hand, Abraham (1998) has presented results for a system in which the same biological evolution equations as in Hernández-Garcia et al. (2002) were used, this time including a maturation time for the zooplankton growth. Such an inclusion gave rise to different small-scale spatial structures for the phytoplankton and the zooplankton, in disagreement with the case of a zero maturation time.

Our main focus in this work is to resolve the discrepancy that arises between the numerical work in Abraham (1998) and the theoretical and numerical work in Hernández-Garcia et al. (2002). In order to compare these two investigations, the same biological model as used by these authors in the case of a non-zero maturation time will be employed. This model belongs to a class of models involving a nutrient, a predator and a prey and represents the interactions between the nutrient, zooplankton and phytoplankton species respectively. These are coupled to the flow by a spatially inhomogeneous forcing. A natural way to characterise the emerging spatial distributions is to investigate the scaling properties of statistical quantities such as Fourier power spectra or structure functions of the corresponding concentration fields. The results of numerical simulations exhibit a small-scale structure that is in good agreement with the results obtained by Neufeld et al. (1999). However, on larger scales there appears to be a decoupling of the spatial structure of zooplankton on the one hand and phytoplankton and nutrient. This result reconciles the contradicting conclusions given by Abraham (1998) and Neufeld et al. (1999).

The paper is organised as follows: in Sect. 2 the mod-

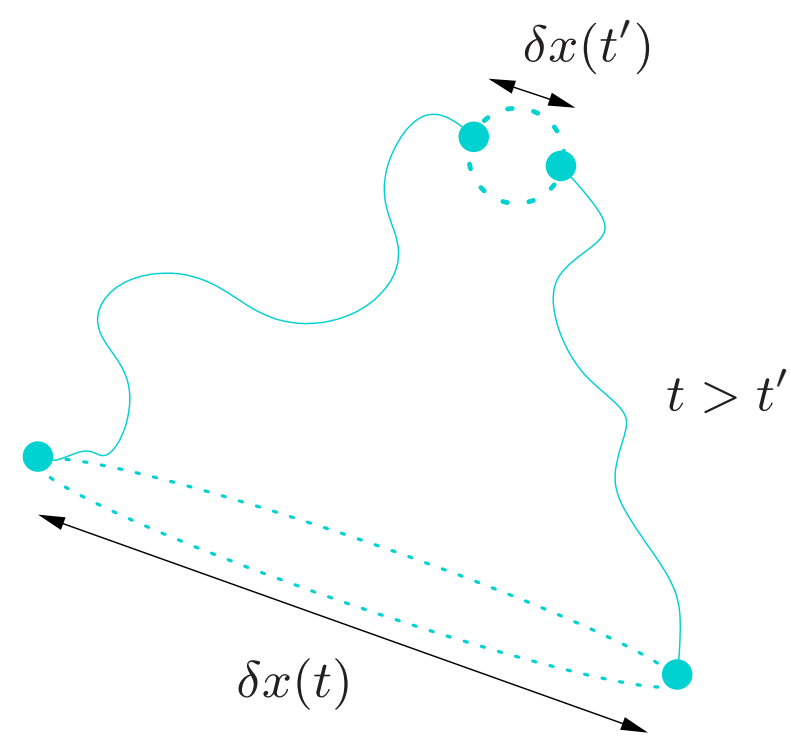

Fig. 1. Set of trajectories for a pair of fluid parcels evolving either forwards $\left(t>t^{\prime}\right)$ or backwards $\left(t<t^{\prime}\right)$ in time. Their separation is dominated by an exponential behaviour such that $\delta x(t) \sim$ $e^{\lambda_{F}\left(t-t^{\prime}\right)} \delta x\left(t^{\prime}\right)$, where $\lambda_{F}$ is the Lyapunov exponent of the flow. The dotted lines represent the stretching of a blob of fluid into a filament by the flow.

els used to describe both the flow and the biology are presented. The numerical scheme employed is explained in Sect. 3 along with the tools used to interpret the results. Section 4 contains the numerical results which illuminate the apparent contradiction between previous results that is the primary focus of this paper. The conclusions are given in Sect. 5.

\section{Biological-fluid coupling}

In the numerical investigations undertaken, we will assume that the flow $\mathbf{v}(\mathbf{x}, t)$ is unsteady, two-dimensional and incompressible. These conditions are usually enough to ensure the chaotic advection of fluid parcels, even if the velocity field is a smooth function of space (Ottino, 1989). A way to characterise the flow is to look at its Lyapunov exponent, $\lambda_{F}$, defined as the exponential rate of separation of initially neighbouring fluid particles (Fig. 1). Since the fluid is incompressible, its volume must be conserved, and therefore contraction must take place in another direction. Moreover, the contraction and also the expansion occur at the same exponential rate $\lambda_{F}$. Consequently, blobs of fluid stretch along long and thin filaments and are repeatedly folded, thus transferring largescale inhomogeneities into smaller ones.

Although the detailed flow used by Abraham (1998) was different, the essential points characterising the strain dominated regions between the eddy regions is captured by the 
flow used here. The domain of the velocity field is taken to be a periodic square with side length $L$, approximately $50 \mathrm{~km}$, corresponding to the characteristic lengthscale of a mesoscale eddy. It will represent the regions that are formed between large eddies, where the phytoplankton filaments are usually observed. This is a pure strain velocity field whose form is

$$
\mathbf{v}(\mathbf{x}, t)=\left[\begin{array}{c}
-\frac{2}{T} \Theta(T / 2-t \quad \bmod T) \cos (2 \pi y / L+\phi) \\
-\frac{2}{T} \Theta(t \quad \bmod T-T / 2) \cos (2 \pi x / L+\theta)
\end{array}\right],
$$

where $\Theta(t)$ is the Heaviside step function defined to be equal to unity for $t \geq 0$ and zero otherwise. The phase angles $\theta$ and $\phi$ change randomly at each period $T$ of the flow, varying the directions of expansion and contraction and hence ensuring all parts of it are equally mixed (Bohr et al., 1998; Ott, 1993). Variation of $T$ has an effect on the magnitude of $\lambda_{F}$ without changing the shape of the trajectories and spatial structure of the flow.

A number of independent fluid parcels are advected by this velocity field. Each one of them carries a uniform distribution of phytoplankton $P$ and zooplankton $Z$, the latter applied mostly to copepod species, as these can be assumed to be drifting with their respective fluid parcels (Abraham, 1998). On the other hand, large zooplankton, such as krill, actively modify their distributions by swimming (Trathan et al., 1993). Moreover, the model described is intended to only represent larger scales (greater than $100 \mathrm{~m}$ or so) on which the flow is quasi two-dimensional. Hence, neglecting any microscopic species motion, e.g. through locomotion or buoyancy, can be justified.

The interactions among the biological species are described in a model already employed by Abraham (1998). This is a typical nutrient-predator-prey system (Murray, 1993), where the former is parametrised by the carrying capacity. This quantity, denoted as $C$, is carried along with the fluid parcel and is defined as the maximum phytoplankton content that the parcel can support in the absence of grazing. As the fluid parcel moves through the domain, the carrying capacity continuously relaxes to a space varying background source, $C_{0}(x, y)=(1-\cos (2 \pi(x+y) / L))$, where $x$ and $y$ are the domain's horizontal and vertical axes respectively. A large scale inhomogeneity is thus introduced into the system.

The species' dynamics are described by the following dimensionless equations,

$$
\begin{aligned}
& \frac{d C}{d t}=\alpha\left(C_{0}(\mathbf{x})-C\right), \\
& \frac{d P}{d t}=P(1-P / C)-P Z, \\
& \frac{d Z}{d t}=P(t-\tau) Z(t-\tau)-\delta Z^{2},
\end{aligned}
$$

where $t$ is the dimensionless time scaled to the phytoplankton production rate $r=0.5 \mathrm{~d}^{-1}(t / r$ is the real time), $\alpha$ denotes the rate at which the carrying capacity relaxes to the background source $C_{0}, \delta$ is the zooplankton mortality rate and $\tau / r$ represents the time taken for the zooplankton to mature. Although it is plausible to assume an instantaneous change in the prey population once prey and predator are encountered, it is not reasonable to assume an instantaneous change in the predator population, and this is the motivation behind the employment of this maturation time.

The phytoplankton growth is logistic and the grazing takes place according to a simple $P Z$ term. In the absence of advection, the parcel position $\mathbf{x}$ remains unchanged. In this case, the model has a single fixed point of equilibrium given by $C^{\star}=C_{0}(\mathbf{x}), P^{\star}=\delta C^{\star} /\left(\delta+C^{\star}\right)$ and $Z^{\star}=P^{\star} / \delta$. This point is a stable fixed point of equilibrium for $\tau=0$, meaning that any perturbation around this point will eventually decay to zero. If $C_{0} \leq 1$ and $\delta \geq 0.5$, as in the simulations performed here, it remains stable for any maturation time.

In the presence of advection, this equilibrium point can never be reached due to the continually varying carrying capacity within the fluid parcel. The continual input of largescale inhomogeneities injected by $C_{0}(\mathbf{x})$ and their transfer to smaller scales by advection leads to a non-trivial statistical steady state. The study of the induced complex patterns will be the focus of the next sections.

\section{Methodology}

The planktonic distributions at a particular time are reconstructed by following an ensemble of fluid parcels. In the method used by Abraham (1998), the fluid parcels are tracked forwards in time and the corresponding distributions are obtained from a Delaunay triangulation of the parcel positions by linear interpolation onto a regular grid. Here, the parcels final positions are fixed to a grid. The parcels are then tracked backwards in time up to a time when their initial biological concentration fields are known. Thereafter, knowing their trajectory, their biological evolution is determined by integrating along this trajectory up to the final time using a second order Runge-Kutta method. This way, no interpolation is necessary and consequently greater accuracy at smaller length scales is achieved. For the concentrations to be accurate up to three decimal places, the timestep is chosen to be 0.001 . This value in real time corresponds to $0.002 \mathrm{~d}$ and is in line with the assumption made of uniformly distributed populations within the fluid parcels. The ensemble of fluid parcels considered here is 250000 and evenly spans a grid of resolution $500 \times 500$. Their initial concentrations are set to be equal to their mean equilibrium values.

A statistical steady state is reached after $20 T$, where $T$ is the period of the flow. The emerging patterns are complex in space (Fig. 3). The standard way to characterise such structures is by their Fourier power spectra. Here, we will also 


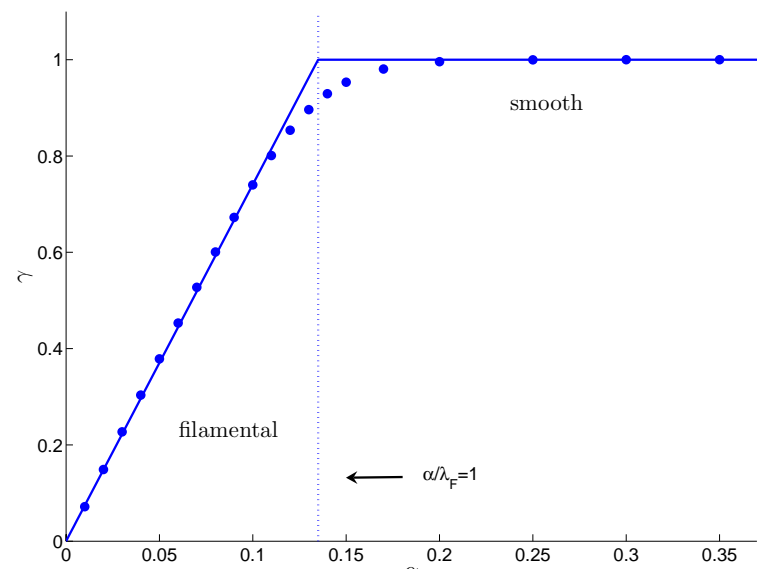

Fig. 2. Variation of the small-scale structure for the phytoplankton carrying capacity in response to the rate $\alpha$, at which it relaxes towards a smoothly varying background source. The structure is characterised by the Hölder exponent, $\gamma$, whose value depends on the ratio of $\alpha$ over the Lyapunov exponent $\lambda_{F}$. When this ratio is bigger than 1 the corresponding structure is smooth otherwise it is filamental. The dots mark $\gamma$ averaged over 500 evenly spaced intersections, while the straight line represents its theoretical value. During this set of experiments, $\lambda_{F} \sim 0.135$.

consider their first-order structure function (Monin and Yaglom, 1975) defined by

$S(\delta x) \equiv\langle|c(x+\delta x)-c(x)|\rangle \sim(\delta x)^{\gamma}$,

where $\langle\ldots\rangle$ denotes averaging over different values of the coordinate $x$. The scaling exponent, $\gamma$, known as the Hölder exponent, is related to the power spectrum exponent, $\beta$, by the simple relationship $\beta=1+2 \gamma$, where $0<\gamma<1$ corresponds to a rough structure and $\gamma=1$ to a smooth one.

\section{Numerical results}

The emergent spatial structures depend on the relative strength of the dispersion of the parcel trajectories and the stability of the biological dynamics. The phytoplankton carrying capacity, whose biological evolution is described by Eq. (2a), has a structure that has been shown in Neufeld et al. (1999) to be characterised by two types of behaviour that depend on the interplay between the relaxation rate $\alpha$ and the Lyapunov exponent $\lambda_{F}$. According to the simplest theory presented in Neufeld et al. (1999), if $\alpha>\lambda_{F}$, the biological processes converge faster to their equilibrium value than the trajectories diverge from each other. The corresponding distribution is smooth. On the other hand, if $\alpha<\lambda_{F}$, the biological processes are too slow to forget the different spatial histories experienced by the parcels. The corresponding distribution is rough, with a Hölder exponent $\gamma=\alpha / \lambda_{F}$ in all directions except for the one that the filaments grow into. This type of structure has been defined by Neufeld et al. (1999)

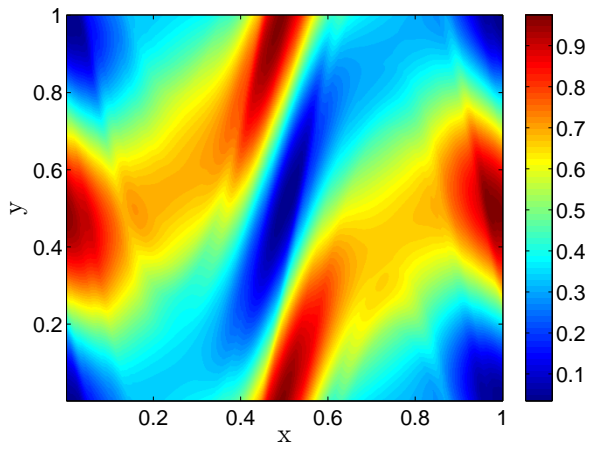

(a) Carrying capacity

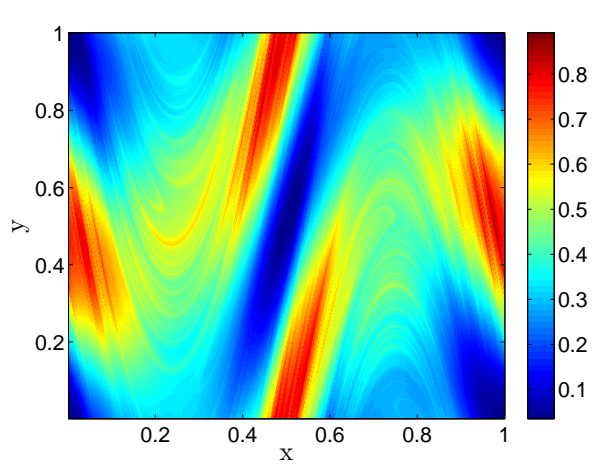

(b) Phytoplankton

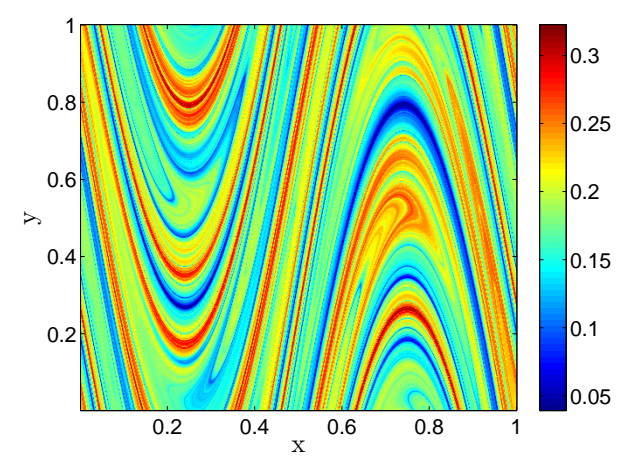

(c) Zooplankton

Fig. 3. Snapshots of the biological distributions at statistical equilibrium $(t=20 T)$. The model follows Eq. (2) with $\tau / r=25 \mathrm{~d}$ and $\delta=2$, denoting a high mortality zooplankton regime. The smoothly varying force $C_{0}(x, y)=(1-\cos (2 \pi(x+y) / L))$ is diagonally orientated. The bar on the right gives the concentration values associated with the different colours. The flow is described in equation 1 where the period $T=20$. The axes are measured in units of $L$, where $L$ is approximately $50 \mathrm{~km}$. 
as filamental. The transition from a filamental to a smooth structure as $\alpha$ varies is depicted in Fig. 2. The numerical agreement with the theoretical prediction gives confidence in the method used here. This is true apart from a small region around $\alpha / \lambda_{F}=1$. The behaviour in this region is not captured by the simple theory presented in Neufeld et al. (1999) and is believed to result from the fact that lengthscales are finite and that there is a distribution of finite time Lyapunov exponents.

As in Abraham (1998), $\alpha$ is taken to be equal to 0.25 corresponding to a tracer that takes $8 \mathrm{~d}$ to adapt to a background force. Choosing a flow with $\lambda_{F} \sim 0.135$ (achieved by setting the period to $T=20$ ), the emerging phytoplankton carrying capacity structure is smooth. This is similar to physical quantities such as the sea surface temperature whose spectral slope has been measured to be $\beta=3$, equivalent to $\gamma=1$ (Deschamps et al., 1981). The limit of $\alpha$ tending to zero corresponds to a tracer that takes an infinite time to adapt to a background source, i.e. a passive non-reactive tracer. Its expected exponent in a two-dimensional turbulent flow is $\beta=1$ or $\gamma=0$ (Powell and Okubo, 1994). The above suggests that although the model considered is simple, it is adequate to describe the transfer of variability to smaller scales and hence capture the basic features of turbulence. Moreover, the exact details of the flow are not important as long as the fluid parcels are chaotically advected.

For a general biological system, the same smooth filamental transition can be obtained. A system similar to the one described in Eq. (2), in the absence of a maturation time $(\tau=0)$, has previously been examined in Hernández-Garcia et al. (2002). In this case, the phytoplankton and zooplankton populations always share the same small-scale structure. This is not the case for the carrying capacity, due solely to it not being symmetrically coupled to the rest of the populations.

Using the same flow as before, a new set of numerical experiments is carried out, with the same biological parameters used by Abraham (1998). Because of the numerical method used, higher spatial resolution can be achieved. Here, the length scales considered reach $0.002 L,(\sim 100 \mathrm{~m}$, the scale at which turbulence ceases to be two-dimensional). The induced spatial patterns can be seen in Fig. 3. At first sight, the phytoplankton and zooplankton populations seem to be decoupled at all length scales, comfirming the picture given by Abraham (1998). However, a transect through the model domain (Fig. 4) shows that at small enough length scales, both phytoplankton and zooplankton exhibit a fine scale structure. Their corresponding power spectra (Fig. 4) reveal that at large wavelengths $\left(k>10 / L \sim 0.2 \mathrm{~km}^{-1}\right)$, they share the same structure with a spectral exponent larger than 1. As expected, the carrying capacity behaves smoothly at all scales. At smaller wavelengths, corresponding to larger length scales, the picture provided by Abraham (1998) is recovered with the phytoplankton spectral slope steepening and the zooplankton one flattening out.

Perhaps a better way to picture this transition is by look-

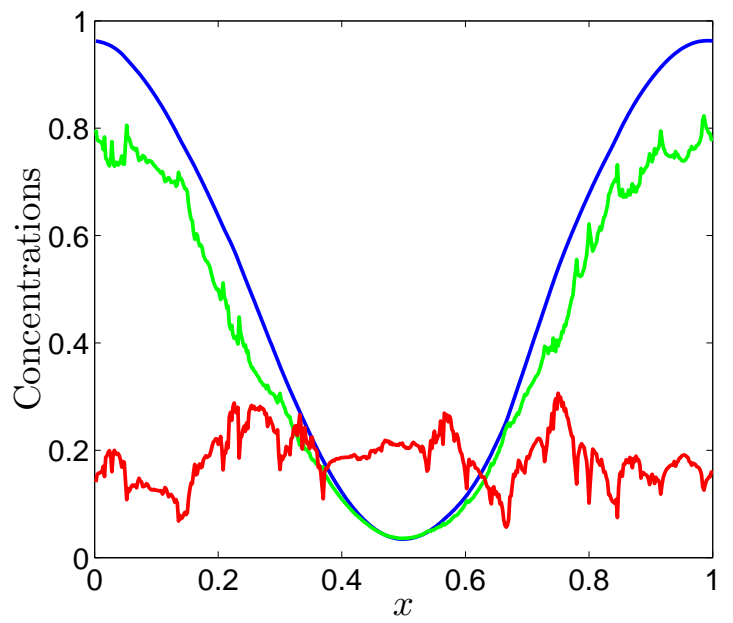

(a) Intersection

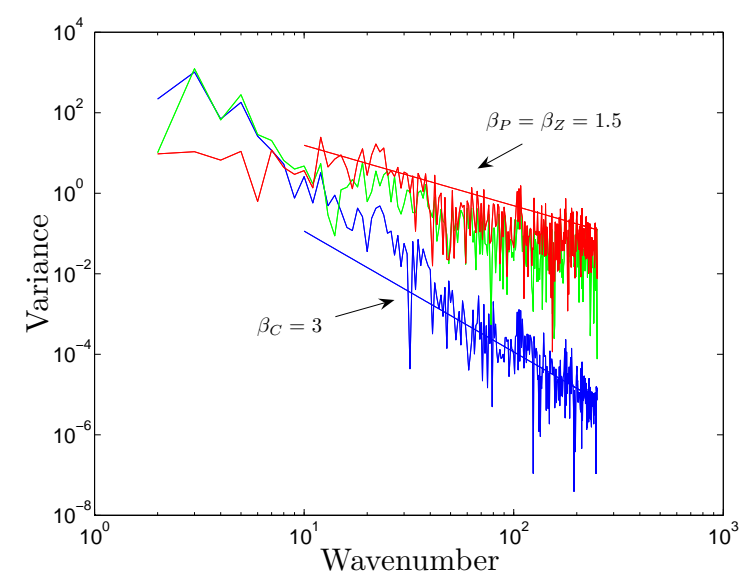

(b) Power Spectrum

Fig. 4. A representative transect (at $y=0.5 L$ ) and the corresponding spectra. Graphs show carrying capacity (blue), phytoplankton (green) and zooplankton (red). The spectra are obtained over 500 evenly spaced horizontal transects and have a power law form. The spectral exponents of the populations are $\beta_{C}=3$ and $\beta_{P}=\beta_{Z}=1.5$. The horizontal axes are measured in units of length $L$ and wavenumber $1 / L$ respectively .

ing at the corresponding first-order structure functions as the length scale $\delta x$ increases (Fig. 5). Initially, the phytoplankton and zooplankton share similar structures. As $\delta x$ increases, there is a regime change where the phytoplankton population starts differentiating from the zooplankton population until it completely decouples it at a characteristic lengthscale $\delta x_{c}$, where $\delta x_{c}$ is approximately equal to $10^{-1} L(\sim 5 \mathrm{~km})$. At this point the phytoplankton acquires a similar distribution to the carrying capacity, while the zooplankton distribution becomes increasingly flat. 


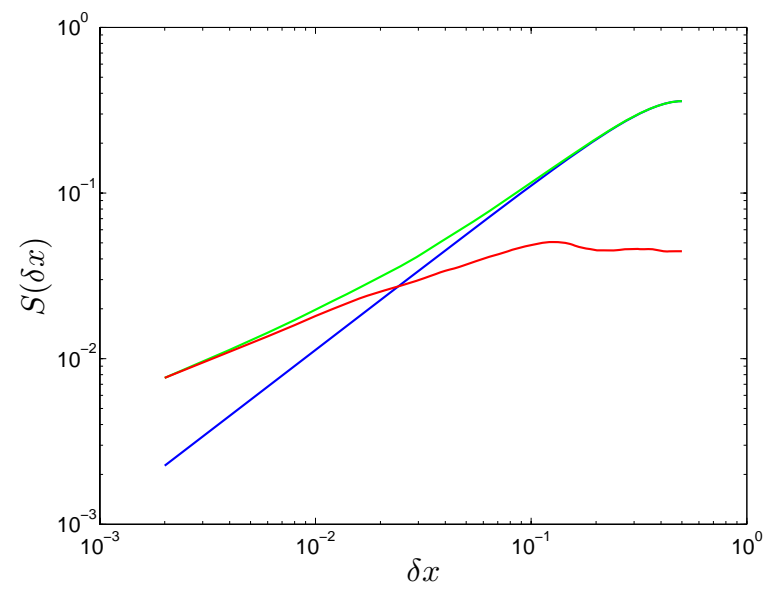

Fig. 5. First-order structure functions averaged over 500 evenly spaced horizontal transects. The graph shows carrying capacity (blue), phytoplankton (green) and zooplankton (red). The horizontal axes are measured in units of length $L$. The Hölder exponent of the carrying capacity is $\gamma_{C}=1$. The phytoplankton and zooplankton respective exponents vary with $\delta x$. For $\delta x<\delta x_{c}$, where $\delta x_{c} \approx 10^{-1} L, \gamma_{P}=\gamma_{Z}=0.5$. For $\delta x>\delta x_{c}, \gamma_{C}=\gamma_{P}=1$ and $\gamma_{Z}=0$.

\section{Conclusions}

The small-scale structure of interacting nutrient, phytoplankton and zooplankton populations, passively advected by a two-dimensional flow and coupled to it through an inhomogeneous source, is here discussed. The particular focus is on the effect induced in these structures by introducing a maturation time in the zooplankton growth.

According to Hernández-Garcia et al. (2002), given a particular class of flow and in the absence of a maturation time, the small-scale structure for the phytoplankton and zooplankton should be the same, given that they are symmetrically coupled, and characterised by a single exponent at all small scales. The inclusion of a maturation time, $\tau$, should not alter the above conclusions. Although the nature of the equations changes from ordinary to delay, there still exists a set of biological decay rates, shared by the phytoplankton and the zooplankton, that should dictate their common structure at small enough scales. This is in disagreement with the numerical results obtained in Abraham (1998). There, the phytoplankton and the carrying capacity turn out to have similar distributions, close to a smooth one and completely decoupled to the zooplankton's. As $\tau$ increases, the zooplankton's distribution becomes increasingly filamental, ultimately behaving like a passive tracer $(\beta \rightarrow 1$ or $\gamma \rightarrow 0)$. The aim of this work has been to resolve this disagreement.

Using a model flow to depict the strain dominated regions formed between mesoscale eddies, we reproduce the regime observed by Abraham (1998). However, the alternative numerical method used here permits the study of smaller length scales where it is revealed that this is only part of the true picture: as long as small enough length scales are considered, a second regime appears where the phytoplankton and zooplankton distributions share the same small-scale structure. The transition between these two regimes occurs at a characteristic lengthscale.

It is important to note that although the biological model under consideration is highly simplified, it can be readily extended by including other species or space-dependent productivity and death rates and these extensions leave the above conclusions unchanged. As long as the biological system remains stable, with a single attractor, the emerging structures will be characterised by a set of spectral exponents or Hölder exponents that will be determined by the competition between the slowest decay rate associated with the biological processes (though when the biological model is spacedependent this decay rate will depend to some extend on the flow) and the Lyapunov exponent associated with the stretching properties of the flow.

By introducing a maturation time, both the phytoplankton and zooplankton structures can no longer be characterised by a single exponent at all scales smaller than that of the flow. Perhaps this point, along with the shared exponent at small enough length scales, should be taken into account in trying to interpret observational measurements in phytoplankton and zooplankton distributions at a large range of lengthscales.

The conditions under which the decoupling of zooplankton and phytoplankton take place are still not completely understood. The effect that the biological and the flow activity have on the characteristic length size of the plankton distributions is the subject of current theoretical investigations that will be reported elsewhere. While many issues remain to be resolved, it is hoped that the present paper will provide another step towards understanding the complicated dynamics of plankton in the presence of oceanic fluid motions.

Edited by: E. Boss

\section{References}

Abraham, E. R.: The generation of plankton patchiness by turbulent stirring, Nature, 391, 577-580, 1998.

Aref, H.: Stirring by chaotic advection, J. Fluid Mech., 143, 1, 1984.

Bohr, T., Jensen, M., Paladin, G., and Vulpiani, A.: Dynamical Systems Approach to Turbulence, Cambridge University Press, Cambridge, 1998

Deschamps, P., Frouin, R., and Wald, L.: Satellite determinations of the mesoscale variability of the sea surface temperature, J. Phys. Oceanogr., 11, 864-870, 1981.

Haynes, P. H.: Transport, stirring an mixing in the atmosphere in Mixing - Chaos and turbulence, edited by: Chate, H., Villermaux, E., and Chomaz, J. M., Kluwer, Dordretch, 1999. 
Hernández-Garcia, E., López, C., and Neufeld, Z.: Spatial patterns in chemically and biologically reacting flows., Proceedings of the 2001 ISSAOS School on Chaos in Geophysical Flows, Otto editore (Torino 2003), 2001.

Hernández-Garcia, E., López, C., and Neufeld, Z.: Small-scale structure of nonlinearly interacting species advected by chaotic flows, CHAOS, 12, 470, 2002.

Kiørboe, T. and Sabatini, M.: 1995 Scaling and fecundity, growth and development in marine planktonic copepods., Mar. Ecol. Prog. Ser., 120, 285-298, 1995.

Klein, P. and Hua, B. L.: The mesoscale variability of the sea surface temperature: An analytical and numerical model., Journal of Marine Research, 48, 729-763, 1990.

Martin, A. P.: Phytoplankton patchiness: the role of lateral stirring and mixing, Progress in Oceanography, 57, 125-174, 2003.

Martin, A. P. and Srokosz, M. A.: Plankton distribution spectra: inter-size class variability and the relative slopes for phytoplankton and zooplankton., Geophys. Res. Lett., 29, 2213, 2002.

McWilliams, J., Weiss, J., and Yavneh, I.: Anisotropy and coherent vortex structures in planetary turbulence, Science, 264, 410-413, 1994.

Monin, A. S. and Yaglom, A. M.: Statistical Fluid Mechanics, MIT Press, Cambridge, 1975.

Murray, J. D.: Mathematical Biology, Springer-Verlag, New York, 1993.
Neufeld, Z., López, C., and Haynes, P. H.: Smooth-Filamental Transition of Active Tracer Fields Stirred by Chaotic Advection, Phys. Rev. Let., 82, 2606, 1999.

Okubo, A.: Oceanic diffusion diagrams, Deep-Sea Res., 18, 802, 1971.

Ott, E.: Chaos in Dynamical Systems, Cambridge University Press, Cambridge, 1993.

Ottino, J. M.: The kinematics of mixing: Stretching, chaos and transport., Cambridge University Press, Cambridge, 1989.

Powell, T. M. and Okubo, A.: Turbulence diffusion and patchiness in the sea, Phil. Trans. R. Soc. Lond. B, 343, 11-18, 1994.

Seuront, L., Schmitt, F., Lagadeuc, Y., Schertzer, D., Lovejoy, S., and Frontier, S.: Multifractal analysis of phytoplankton biomass and temperature in the ocean., Geophys. Res. Lett., 23, 35913594, 1996.

Seuront, L., Schmitt, F., Lagadeuc, Y., Schertzer, D., and Lovejoy, S.: Universal multifractal analysis as a tool to characterize multiscale intermittent patterns: example of phytoplankton distribution in turbulent coastal waters., J. Plankton Res., 21, 977-922, 1999.

Trathan, P. N., Priddle, J., Watkins, J. L., Miller, D. G. M., and Murray, A. W. A.: Spatial variabiity of Antarctic krill in relation to mesoscale hydrography., Mar. Ecol. Prog. Ser., 98, 61-71, 1993.

Tsuda, A., Sugisaki, H., Ishimoru, T., Saino, T., and Sato, T.: White-noise-like distribution of the oceanic copepod Neocalanus cristatus in the subarctic North Pacific., Mar. Ecol. Prog. Ser., 97, 39-46, 1993. 